\title{
181. The Tilting of the Surface of a Semi-infinite Solid due to the Internal Nuclei of Strain.
}

\author{
By Katsutada SezAwA. \\ Earthquake Research Institute, Tokyo Imperial University. \\ (Rec. Nov. 20, 1928. Comm. by K. SuYehiro, M.I.A., Dec. 2, 1928.)
}

The present problem may have some importance on geophysics in that the clue to the internal state of the earth crust may be obtained from the surface data.

We shall take the free surface of the semi-infinite solid to be $z=0$, and also take that the positive sense of the axis of $z$ is directed towards the interior of the body. In a three-dimensional problem the effect of the symmetrical nucleous, resident at the point $r=0, z=\xi$, can be expressed in the form:

$$
\lrcorner_{0}=1 / \sqrt{r^{2}+(z-\xi)^{2}},
$$

the corresponding displacement, which fulfils the conditions of symmetry at the nucleous, being easily formulated. Again, the conditions on the free surface are satisfied by considering the image and afterwards superposing on the primary deformation some free ${ }^{1)}$ displacements which are accumulated in the neighbourhood of the surface.

Thus, we find the expressions of the horizontal and the vertical components of the displacement of the surface of the body in the form:

$$
\begin{aligned}
& u=-\int_{0}^{\infty}\left[\frac{(\lambda+2 \mu)^{2}}{\mu(\lambda+\mu)} \frac{1}{f^{2}}+\frac{(\lambda+\mu)}{\mu} \frac{\xi}{f}\right] e^{-f \xi} \frac{\partial J_{0}(f r)}{\partial r} d f, \\
& w=-\int_{0}^{\infty}\left[\frac{(\lambda+2 \mu)}{(\lambda+\mu)} \frac{1}{f}+\frac{(\lambda+2 \mu)}{\mu} \xi\right] e^{-f \xi} J_{0}(f r) d f .
\end{aligned}
$$

The tilting of the surface can be easily expressed by

$$
\frac{\partial w}{\partial r}=\frac{(\lambda+2 \mu)}{(\lambda+\mu)}\left(\frac{\sqrt{\xi^{2}+r^{2}}-\xi}{r \sqrt{\xi^{2}+r^{2}}}\right)+\frac{(\lambda+2 \mu)}{\mu} \frac{\xi r}{\left(\xi^{2}+r^{2}\right)^{\frac{3}{2}}} .
$$

1) Prof. K. Terazawa, J. Coll. Sci., Tokyo, 37 (1916). 
No. 10.] The Tilting of the Surface of a Semi-infinite Solid due.

Proceeding in similar manners, we can determine the effect of the nucleous in the form of a horizontal doublet such that

$$
J_{0}=\frac{\partial}{\partial x} \frac{1}{\sqrt{r^{2}+(z-\xi)^{2}}}
$$

The surface displacement in this case is expressed by

$$
\begin{aligned}
u & =\cos \omega \int_{0}^{\infty}\left[\frac{(\lambda+2 \mu)^{2}}{\mu(\lambda+\mu)} \frac{1}{f}+\frac{\lambda+\mu}{\mu} \xi\right] e^{-f^{\xi}} \frac{\partial J_{1}(f r)}{\partial r} d f, \\
-v & =\sin \omega \int_{0}^{\infty}\left[\frac{(\lambda+2 \mu)^{2}}{\mu(\lambda+\mu)} \frac{1}{f}+\frac{\lambda+\mu}{\mu} \xi\right] e^{-f^{\xi}} \frac{J_{1}(f r)}{r} d f, \\
w & =\cos \omega \int_{0}^{\infty}\left[\frac{(\lambda+2 \mu)}{(\lambda+\mu)}+\frac{(\lambda+2 \mu)}{\mu} f \xi\right] e^{-f \xi} J_{1}(f r) d f,
\end{aligned}
$$

in which $u, v, w$ are radial, azimuthal and vertical components of the displacement on $z=0$. The radial and the azimuthal tiltings of the surface are of the form :

$$
\begin{gathered}
\frac{\partial w}{\partial r}=\cos \omega\left[\frac{(\lambda+2 \mu)}{(\lambda+\mu)}\left(-\frac{1}{r^{2}}+\frac{\xi}{r^{2} \sqrt{\xi^{2}+r^{2}}}+\frac{\xi}{\left(\xi^{2}+r^{2}\right)^{3}}\right)\right. \\
\left.+\frac{(\lambda+2 \mu)}{\iota^{\prime}}\left(\frac{\xi}{\left(\xi^{2}+r^{2}\right)^{\frac{3}{2}}}-\frac{3 \xi r^{2}}{\left(\xi^{2}+r^{2}\right)^{\frac{5}{2}}}\right)\right], \\
-\frac{1}{r} \frac{\partial w}{\partial \omega}=\sin \omega\left[\frac{(\lambda+2 \mu)}{(\lambda+\mu)}\left(\frac{\sqrt{\xi^{2}+r^{2}}-\xi}{r^{2} \sqrt{\xi^{2}+r^{2}}}\right)+\frac{(\lambda+2 \mu)}{\mu} \frac{\xi}{\left(\xi^{2}+r^{2}\right)^{\frac{3}{2}}}\right] .
\end{gathered}
$$

When the nucleous is a vertical doublet of the type,

$$
\Delta_{0}=\frac{\partial}{\partial z} \frac{1}{\sqrt{r^{2}+(z-\xi)^{2}}}
$$

the surface displacement and the tilting are expressed by

$$
\begin{aligned}
& u=\int_{0}^{\infty}\left[\frac{(\lambda+2 \mu)^{2}}{\mu(\lambda+\mu)} \frac{1}{f}+\frac{(\lambda+\mu)}{\mu} \xi\right] e^{-f^{\xi}} \frac{\partial J_{0}(f r)}{\partial r} d f, \\
& w=\int_{0}^{\infty}\left[\frac{(\lambda+2 \mu)}{(\lambda+\mu)}+\frac{(\lambda+2 \mu)}{\mu} f \xi\right] e^{-f \xi} J_{0}(f r) d f,
\end{aligned}
$$




$$
\frac{\partial w}{\partial r}=-\left[\frac{(\lambda+2 \mu)}{(\lambda+\mu)} \frac{r}{\left(\xi^{2}+r^{2}\right)^{\frac{3}{2}}}+\frac{3(\lambda+2 \mu)}{\mu} \frac{\xi^{2} r}{\left(\xi^{2}+r^{2}\right)^{\frac{5}{2}}}\right]
$$

The author has taken up three kinds of nuclei, namely the symmetrical type, ii) the horizontal doublet and iii) the vertical doublet; the ratio of the surface-tilting to the strain in the vicinity of each nucleous is illustrated in Figs. 1, 2 and 3, Poisson's ratio being taken to be $\frac{1}{4}$ in each case. Discussions on these results are omitted in the present paper.

The detailed explanation of this problem and also the treatment of similar problems, such as the effects of multiplet-nuclei and of more complex nuclei having some geometrical forms, both in two- and threedimensions, will be published in the Bull. VII of the Earthquake Research Institute.

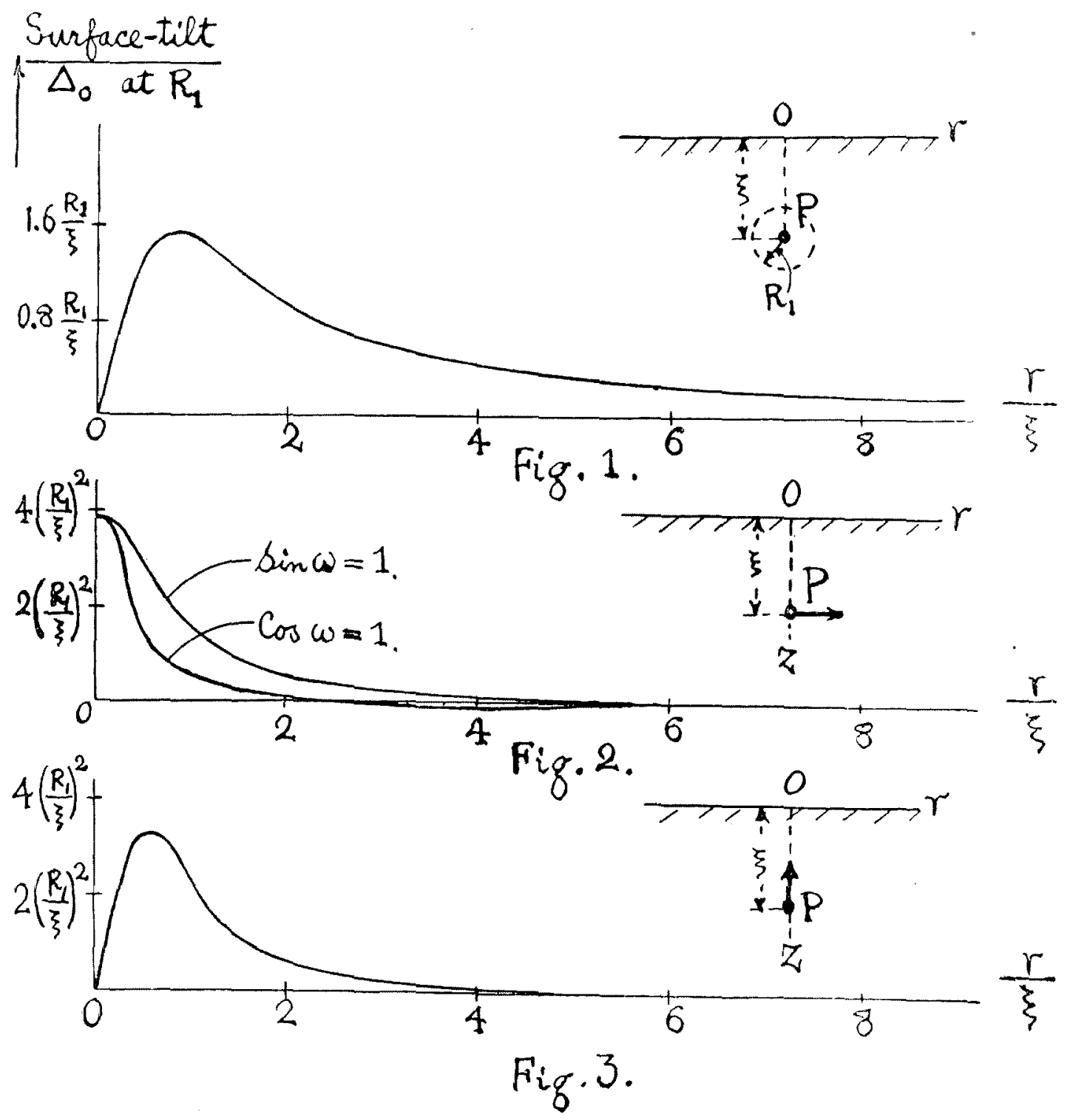

